\title{
Gauss Quadratures and Jacobi Matrices for Weight Functions Not of One Sign
}

\author{
By J. Kautsky and S. Elhay
}

\begin{abstract}
Construction of Gauss quadratures with prescribed knots via Jacobi matrices is extended to the case where not all orthogonal polynomials exist due to the weight function changing sign. An algorithm is described and is demonstrated by calculating the knots of Kronrod schemes and other Gauss quadratures with prescribed knots.
\end{abstract}

1. Introduction. Let $w$ be a real-valued function on some (finite, semi-infinite or infinite) real interval $(a, b)$ such that $I(f):=\int_{a}^{b} f(t) w(t) d t$ exists for any polynomial $f$. The quadrature formula

$$
Q(f):=\sum_{j=1}^{n} \sum_{i=1}^{n_{j}} C_{j i} f^{(i-1)}\left(x_{j}\right)+\sum_{j=1}^{m} \sum_{i=1}^{m_{j}} D_{j i} f^{(i-1)}\left(v_{j}\right)
$$

using the fixed (or prescribed) knots $v_{1}, v_{2}, \ldots, v_{m}$ of multiplicities $m_{1}, m_{2}, \ldots, m_{m}$ is said to be Gaussian if the weights $\left(C_{j i}\right.$ and $\left.D_{j i}\right)$ and the free (or Gauss) knots $x_{1}, x_{2}, \ldots, x_{n}$, of multiplicities $n_{1}, n_{2}, \ldots, n_{n}$, are chosen so that $I(f)=Q(f)$ for all $f$ a polynomial of order $N+n$ or less. Here $N:=\sum_{j=1}^{n} n_{j}+\sum_{j=1}^{m} m_{j}$ is the number of weights in the quadrature. The following characterization and sufficient condition for the existence of the Gauss knots are well known (see, e.g., Turan [11]).

TheOREM 1. Let $q_{F}(t):=\prod_{j=1}^{m}\left(t-v_{j}\right)^{m_{j}}$ and $q_{G}(t):=\prod_{j=1}^{n}\left(t-x_{j}\right)^{n_{j}}$. The knots $x_{1}, x_{2}, \ldots, x_{n}$ are Gauss knots if and only if

$$
\int_{a}^{b} t^{k} q_{G}(t) q_{F}(t) w(t) d t=0
$$

for $k=0,1, \ldots, n-1$.

THEOREM 2. If $w \geqslant 0$ on $(a, b)$, if the multiplicities $n_{j}$ of the Gauss knots are odd, and if the multiplicities $m_{j}$ of the fixed knots $v_{j} \in(a, b)$ are even, then there exist real and distinct Gauss knots $x_{j} \in(a, b), j=1,2, \ldots, n$.

In [2] Golub and Kautsky present a method which stably and efficiently determines the Gauss knots of a quadrature satisfying the condition in Theorem 2. Their method hinges on the fact that associated with every $w \geqslant 0$ there exists a (tridiagonal) Jacobi matrix the elements of which are the coefficients in the three-term recurrence satisfied by the polynomials orthogonal on $(a, b)$ with respect to $w$. The

Received August 23, 1983; revised January 19, 1984.

1980 Mathematics Subject Classification. Primary 65F15, 65D30. 
eigenvalues of the principal submatrices of this matrix are the zeros of the polynomials and so they are the knots of the Gauss quadratures. In particular, knowing the Jacobi matrix $J$ for a given $w \geqslant 0$, they show how to find the Jacobi matrix $\hat{J}$ for $\hat{w}(t):=\sigma(t-v)^{s} w(t), s=1,2$ (where $\sigma$ is a constant chosen to achieve $\hat{w} \geqslant 0$ on $[a, b])$ and then find the Jacobi matrix for the weight function

$$
\hat{w}:=q_{F}(t) w(t),
$$

where $q_{F}(t)$ is a product of such factors, by repeating the process. The required Gauss knots are then easily found from $\hat{J}$.

In this paper we aim to extend results of [2] to the case of simple knots prescribed inside the interval $(a, b)$. In [6] Kronrod computed, for the case of the constant weight function, the quadratures using $2 n+1$ simple knots of which $n$ are the zeros of the $n$th degree Legendre polynomial and the other $n+1$ are Gaussian $(n \leqslant 40)$. Patterson [9] later extended these quadratures by adding free knots to quadratures which have the Kronrod knots prescribed. In both [6] and [9] the quadratures were computed by determining the required polynomials from the moments of the weight function and then solving for their roots. Patterson improved Kronrod's method of determining the polynomials by expressing them as linear combinations of Legendre polynomials. Piessens and Branders [10] used Chebyshev rather than Legendre polynomials to further stabilize the process. A recent survey of theoretical and numerical results concerning such quadratures appears in [8].

When simple knots are prescribed inside the interval, the assumptions of Theorem 2 are no longer satisfied. The existence and the properties of the required quadratures depend on polynomials orthogonal with respect to a weight function which changes sign inside the interval. For some degrees such polynomials may not exist or may have zeros which are multiple, outside $(a, b)$ or even complex. Consequently, the corresponding quadratures may not exist or may have knots and weights which render them useless.

To apply the techniques of [2] to such quadratures we generalize the concept of Jacobi matrices for nonnegative weight functions - when orthogonal polynomials of all degrees exist - to the situation where only some orthogonal polynomials may exist. For this purpose, to replace the Jacobi matrix, we introduce in Section 2 a pair of matrices-a recurrence matrix of the polynomials and the Gram matrix which measures their orthogonality. In Section 3, we show the relation between such pairs of matrices for two different weight functions, particularly those differing by a linear factor. This is the basis for a numerical procedure described and tested in Sections 4 and 5.

2. Generalizing Jacobi Matrices for Arbitrary Weight Functions. In this section we introduce the recurrence and Gram matrices for a given weight function and establish some basic relations. We will deal with real functions of a real variable only. Let $k \geqslant 1$ be an integer and let $\mathbf{p}:=\left[p_{0}(t), p_{1}(t), \ldots, p_{k-1}(t)\right]^{T}$ and $p_{k}(t)$ be a set of monic (leading coefficient equal unity) polynomials, so that $p_{j}(t)$ has exact degree $j$. There exists a unique lower Hessenberg matrix $K$, with all elements in the super-diagonal equal unity, such that

$$
t \mathbf{p}(t)=K \mathbf{p}(t)+p_{k}(t) \mathbf{e}_{k} .
$$


Here, as later, $\mathbf{e}_{k}$ is the $k$ th column of an identity matrix with appropriate dimension. We shall call $K$ the recurrence matrix for the polynomials $p_{j}(t)$ because its elements define a recurrence relation by which $p_{j}(t)$ can be found from its predecessors.

Since $p_{0}(t)=1$, we have $\mathbf{p}(t) \neq \mathbf{0}$ for any $t$ and thus, from (2.1), any zero $v$ of $p_{k}(t)$ is an eigenvalue of $K$.

Let $w$ be a weight function defined on $(a, b)$. We will denote by $M$ the symmetric Gram matrix

$$
M:=\int_{a}^{b} \mathbf{p p}^{T} w d t
$$

A simple calculation with (2.1) shows that

$$
K M=\int_{a}^{b} t \mathbf{p p}^{T} w d t-\mathbf{e}_{k} \mathbf{b}^{T}
$$

where

$$
\mathbf{b}:=\int_{a}^{b} p_{k} \mathbf{p} w d t .
$$

Clearly $K M$ is a symmetric matrix plus a rank-one correction to its last row. We may note here that the vector $\mathbf{b}$ is therefore determined by the matrices $K$ and $M$ up to its last element and we have

$$
\mathbf{b}-\mathbf{b}^{T} \mathbf{e}_{k} \mathbf{e}_{k}=\left(K M-M K^{T}\right) \mathbf{e}_{k} .
$$

The polynomial $p_{k}$ is orthogonal to $p_{0}, p_{1}, \ldots, p_{k-1}$ with respect to $w$ iff $\mathbf{b}=\mathbf{0}$; furthermore, when the polynomials $p_{0}, p_{1}, \ldots, p_{k}$ are all orthogonal with respect to $w$, then, in addition, $M$ is diagonal and since $K M$ is lower Hessenberg and symmetric, $K$ is tridiagonal. Its elements are the coefficients in the well-known three-term recurrence for orthogonal polynomials. If the orthogonal polynomials are normalized, then $M$ is the identity and $K$ is called the Jacobi matrix for $w$.

As we are interested in the case where orthogonal polynomials of all orders need not exist, the matrix $M$ may no longer be diagonal. However, we observe that a particular polynomial $p_{j-1}(t)$ is orthogonal, with respect to $w$, to all polynomials of lesser degree if and only if the last row and column of the $j \times j$ principal submatrix of $M$ can have a nonzero element only on the diagonal. For convenience, we will call such a matrix $\left(M:=\left[m_{r s}\right]\right.$ with $\left.m_{r j}=m_{j r}=0, r=1,2, \ldots, j-1\right) j$-diagonal. The eigenvalues of the corresponding $(j-1) \times(j-1)$ principal submatrix of $K$ will be the zeros of $p_{j-1}(t)$.

Thus the association between a sequence of polynomials orthogonal with respect to a nonnegative weight function and the Jacobi matrix can be generalized to an association between any sequence of monic polynomials, an arbitrary (suitably integrable) weight function and a pair of matrices $K, M$.

3. Modifying Generalized Jacobi Matrices. Let $v$ be a real scalar that may or may not lie in $(a, b)$. We show in the next section how to determine the matrices $\hat{K}$ and $\hat{M}$ for the weight function $\hat{w}(t):=(t-v) w(t)$ from the matrices $K$ and $M$. Repeated application of the process will give the $\hat{K}$ corresponding to the weight function $\hat{w}$ of the type (1.2) for $q_{F}$ a product of such factors. Algorithms of this type have been implemented in [1] and [2] for the case of prescribed knots preserving the 
nonnegativity of the weight function. We note that from (2.3) it follows that the left-hand side of

$$
(K-v I) M=\int_{a}^{b}(t-v) \mathbf{p p}^{T} w d t-\mathbf{e}_{k} \mathbf{b}^{T}
$$

is again a symmetric matrix plus a rank-one correction to its last row. This leads to the following result.

THEOREM 3. Let the monic polynomials $p_{0}, p_{1}, \ldots, p_{k}$ have recurrence matrix $K$, let $L$ be a unit lower triangular matrix, and $\mathbf{c}$ be some vector. Then the polynomials $\hat{p}_{0}, \hat{p}_{1}, \ldots, \hat{p}_{k}$ defined by

$$
\begin{gathered}
\hat{\mathbf{p}}:=L^{-1} \mathbf{p}, \\
\hat{p}_{k}:=p_{k}-\mathbf{c}^{T} \hat{\mathbf{p}}
\end{gathered}
$$

are monic and their recurrence matrix $\hat{K}$ satisfies

$$
\hat{K}=L^{-1} K L+\mathbf{e}_{k} \mathbf{c}^{T} .
$$

If, in addition, $v$ is any scalar, $\hat{w}(t):=(t-v) w(t), M$ is as in (2.2) and we similarly define $\hat{M}$ by

$$
\hat{M}:=\int_{a}^{b} \hat{\mathbf{p}}^{T} \hat{w} d t
$$

then

$$
(K-v I) M=L \hat{M} L^{T}-\mathbf{e}_{k} \mathbf{b}^{T},
$$

with $\mathbf{b}$ as in (2.4), and, if $M$ is nonsingular, then we also have

$$
\hat{K}=v I+\hat{M} L^{T} M^{-1} L-\mathbf{e}_{k}\left(\mathbf{b}^{T} M^{-1} L-\mathbf{c}^{T}\right) .
$$

Proof. Relation (3.4) follows immediately by substituting (3.2) and (3.3) into (2.1) and noting that $L \mathbf{e}_{k}=\mathbf{e}_{k}$; similarly (3.6) is obtained from (3.1) and (3.2). Now from (3.4) it follows, if $M$ is nonsingular, that

$$
\hat{K}-v I=L^{-1}(K-v I) M M^{-1} L+\mathbf{e}_{k} \mathbf{c}^{T}=L^{-1}\left(L \hat{M} L^{T}-\mathbf{e}_{k} \mathbf{b}^{T}\right) M^{-1} L+\mathbf{e}_{k} \mathbf{c}^{T}
$$

from which we have (3.7).

The first part of Theorem 3 gives in (3.4) the relation between recurrence matrices of any two (monic) polynomial bases - the weight functions are not involved here. Given $K, M$ (representing the polynomial base $\mathbf{p}$ and $w$ ) and $v$ there are many pairs $\hat{K}, \hat{M}$ representing some other base $\hat{\mathbf{p}}$ and $\hat{w}$; the second part of the theorem gives relations (3.6) and (3.7) (or (3.4)) which every such pair must satisfy. Any choice of $L$ and $\hat{M}$ satisfying (3.6) determines the pair $\hat{K}, \hat{M}$ uniquely. We show in the next section how to construct a unit lower triangular $L$ and all but the last diagonal element of $\hat{M}$ in (3.6), without using b. This in turn uniquely determines all but the last row of $\hat{K}$. Furthermore, by (3.4) the matrix $\hat{K}-\mathbf{e}_{k} \mathbf{c}^{T}$, which differs from $\hat{K}$ only in its last row, is similar to $K$ and has the property that, although all its eigenvalues are those of $K$, the eigenvalues of its principal $j \times j$ submatrix (each $j<k$ ) are the zeros of $\hat{p}_{j}$. Consequently a $\hat{K}$ matrix of dimension $n$ can be found by constructing $\hat{K}$ of dimension $k:=n+1$ and then discarding the last row and column.

Thus the important so-called de-escalation property, which was already observed in [2] for the construction of modified Jacobi matrices and which avoids the explicit determination of vectors $\mathbf{c}$ and $\mathbf{b}$, is preserved. 
Finally, we observe that Theorem 3 is a straightforward generalization of Theorem 3 of [2] to the case where $M$ and $\hat{M}$ need no longer be diagonal. In particular, the "symmetric $L R$ transformation with shift $v$ " of [2] is here replaced by relations (3.6) and either (3.4) or (3.7) which now include the Gram matrices $M$ and $\hat{M}$.

4. Derivation of the Algorithm. Here we derive an explicit procedure which, given matrices $K$ and $M$ for some weight function $w$, and a scalar $v$ will produce the matrices $\hat{K}$ and $\hat{M}$ for the weight function $\hat{w}(t):=(t-v) w(t)$. As pointed out earlier, such a pair is not unique and this fact is reflected by the nonuniqueness of the factoring (3.6) in Theorem 3 where we use $\frac{1}{2} n(n+1)-1$ elements from the left-hand side to determine $n^{2}$ elements on the right-hand side. Blocking the matrices in (3.6) as shown in Figure 1 with a similar blocking for $\hat{M}$, we have

$$
\begin{gathered}
Y_{j}=L_{j} \hat{M}_{j} L_{j}^{T}, \\
L_{j}\left(\hat{M}_{j} \ell_{j}+\hat{\mathbf{m}}_{j}\right)=\mathbf{y}_{j},
\end{gathered}
$$

and

$$
a_{j}+\delta_{j k} \mathbf{b}^{T} \mathbf{e}_{k}=\ell_{j}^{T}\left(\hat{M} \ell_{j}+2 \hat{\mathbf{m}}_{j}\right)+\hat{d}_{j}
$$

Thus the matrices $L$ and $\hat{M}$ can be built up one row and column at a time provided we choose $\ell_{j}$ and $\hat{\mathbf{m}}_{j}$ satisfying (4.2). Our aim is to construct an $\hat{M}$ which is $i$-diagonal for as many $i=1,2, \ldots, k$ as feasible. Consequently we must set $\hat{\mathbf{m}}_{j}=\mathbf{0}$ in (4.2) if possible. This can always be done when $\hat{M}_{j}$ is nonsingular and $\ell_{j}$ will then be the solution of

$$
\hat{M}_{j} \ell_{j}=\mathbf{z}_{j}:=L_{j}^{-1} \mathbf{y}_{j} .
$$

For singular $\hat{M}_{j}$ we propose the following two schemes (any $\ell_{j}$, $\hat{\mathbf{m}}_{j}$ satisfying (4.2) would, of course, do):

Scheme (a). Set $\ell_{j}:=\mathbf{0}$ and $\hat{\mathbf{m}}_{j}=\mathbf{z}_{j}$.

Scheme (b). Choose any least squares solution $\ell_{j}$ of (4.4) and set $\hat{\mathbf{m}}_{j}$ to the residual

$$
\hat{\mathbf{m}}_{j}:=\mathbf{z}_{j}-\hat{M}_{j} \ell_{j} \text {. }
$$

We note that Scheme (a) chooses $\hat{p}_{j}:=p_{j}$ (and $L(j+1)$-diagonal) while Scheme (b) aims to reduce the sum of squares of the off-diagonal elements of the Gram matrix $\hat{M}$. Furthermore, the Scheme (b) can be used regardless of the singularity of $\hat{M}_{j}$-it will always produce a solution of (4.4) if one exists. From the relations (4.1)-(4.3) it

$$
\begin{aligned}
& (K-v I) M \quad \mathbf{e}_{k} \mathbf{b}^{T} \\
& {\left[\begin{array}{cc}
Y_{j} & \mathbf{y}_{j} \cdot \\
\mathbf{y}_{j}^{T} & a_{j} \\
\cdot & \cdots
\end{array}\right]+\left[\begin{array}{ccc}
0 & \cdots & 0 \\
\cdot & & \cdot \\
0 & \cdots & 0 \\
\cdot & \mathbf{b}^{T} & \cdot
\end{array}\right]} \\
& L \quad \hat{M} \quad L^{T}
\end{aligned}
$$

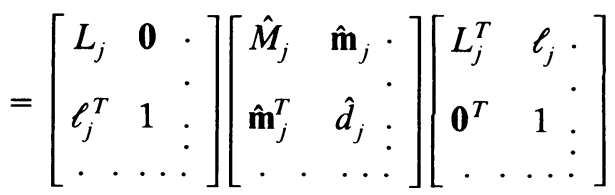

FIGURE 1

Blocking of Eq. (3.6). Matrices $Y_{j}, L_{j}$ and $\hat{M}_{j}$ have order $j$ 
is obvious that we can determine the whole of $L$ and all but the element $\hat{d}_{k}$ of $\hat{M}$ from $Y_{k}$ (i.e., from $K, M$ and $v$ ). This is important because $\hat{\mathbf{m}}_{k}=\mathbf{0}$ indicates the orthogonality of $\hat{p}_{k-1}$ the zeros of which are the eigenvalues of the de-escalated $\hat{K}$.

We have implemented an algorithm performing a sequence of such steps corresponding to fixed knots $v:=v_{j}, j=1,2, \ldots$ repeated according to their multiplicities. The algorithm begins with matrices of sufficient size and de-escalates them by discarding one row and column at a time after modifying them by each linear factor. We use the column pivoted $Q R$ decomposition of $\hat{M}_{j}$ to determine its singularity in Scheme (a) or to find the least squares solution in Scheme (b). In the latter case this will provide $\ell_{j}$ with at least as many zeros as is the rank deficiency of $\hat{M}_{j}$.

The new recurrence matrices are computed by (3.4) although no essential difference has been observed when (3.7) was used instead. The detailed algorithm can be found in [4].

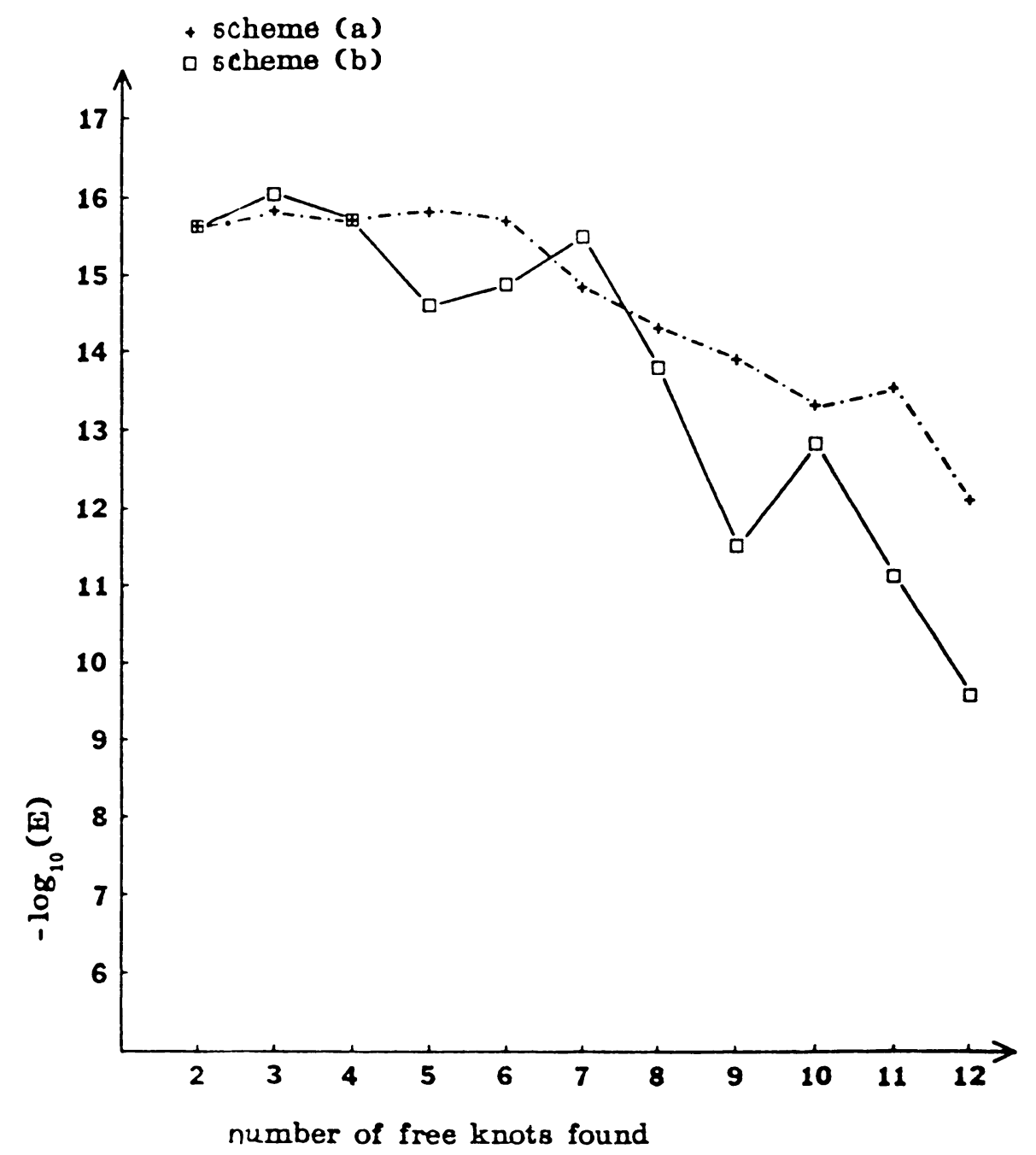

Figure 2

Error $E$ in calculating the knots of Kronrod quadratures

by $(+)$-scheme (a) and ( $\square)$-scheme (b). 


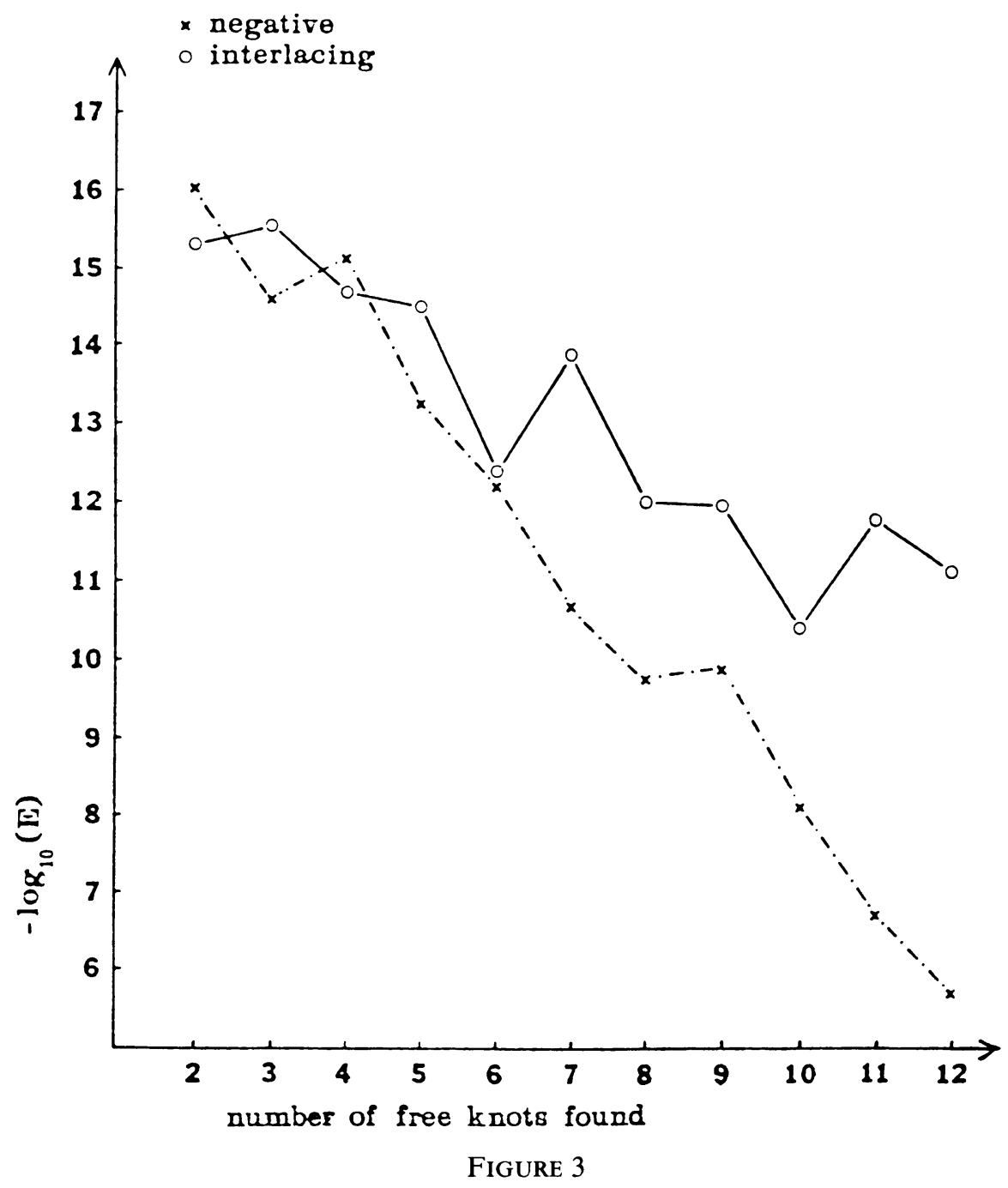

Error $E$ in calculating $N / 2$ free knots where the $N / 2$ are alternate $\left({ }^{\circ}\right)$ or negative $(\times)$ zeros of the Legendre polynomial of degree $N$. For this test schemes (a) and (b) give identical results.

5. Numerical Tests. In this section we present the results of numerical experiments which serve to demonstrate the methods of the previous section.

Firstly, we have recomputed the knots of some of the Kronrod quadratures in [6]. Here the zeros of the Legendre polynomial of degree $n$ are prescribed and $n+1$ free knots are calculated. The maximum (absolute) difference between the results of Kronrod's calculations and those obtained by our Schemes (a) and (b) are shown in Figure 2 (on negative logarithmic scale) as a function of the number of knots computed.

Secondly, we have designed the following test. We compute the zeros of the $N$-degree Legendre polynomials as the eigenvalues of the corresponding Jacobi matrix. We then select $k<N$ of these as fixed knots, calculate $N-k$ free knots by our methods, and compare them with the remaining zeros. In Figure 3, we show the results for the cases where we prescribe $k=[N / 2]$ zeros either interlacing the free 
knots to be found or those in $(-1,0)$. While the former choice of prescribed knots resembles the Kronrod scheme, the latter by its unbalanced nature appears to provide a severe test for the methods.

All results were computed using the MATLAB package [7] on a VAX 11/750 under the VMS operating system in double (56-bit mantissa) precision.

We should emphasize that these tests were designed only to demonstrate the viability of the methods of the previous section and no serious attempt was made to exploit the special structure of the matrices involved in the process. We believe that taking advantage of this special structure will lead to an efficient numerical procedure based on this approach.

Acknowledgement. The authors wish to thank the referee for useful comments and suggestions.

School of Mathematical Sciences

Flinders University of South Australia

Bedford Park, S.A. 5042, Australia

Department of Computer Science

University of Adelaide

G.P.O. Box 498

Adelaide, S.A. 5001, Australia

1. W. GAUTSCHI, "An algorithmic implementation of the generalized Christoffel theorem," in Numerical Integration, ISNM 57 (G. Hämmerlin, ed.), Birkhäuser, Basel, 1982, pp. 89-106.

2. G. H. Golub \& J. KaUTSKY, "Calculation of Gauss quadratures with multiple free and fixed knots," Numer. Math., v. 41, 1983, pp. 147-163.

3. G. H. Golub \& J. H. WelSCH, "Calculation of Gauss quadrature rules," Math. Comp., v. 23, 1969, pp. 221-230.

4. J. KaUtsky \& S. Elhay, Gauss Quadratures and Jacobi Matrices for Weight Functions Not of One Sign, Report, School of Math. Sci., Flinders University of South Australia, 1983.

5. J. KAUTSKY \& G. H. GoluB, “Evaluation of Jacobi matrices," Linear Algebra Appl., v. 52/53, 1983, pp. 439-455.

6. A. S. Kronrod, Nodes and Weights for Quadrature Formulae. Sixteen Place Tables, "Nauka", Moscow, 1964; English transl., Consultants Bureau, New York, 1965.

7. C. Moler, MATLAB User's Guide, Dept. of Comput. Sci., University of New Mexico, 1981.

8. G. MONEGATO, "Stieltjes polynomials and related quadrature rules," SIAM Rev., v. 24, 1982, pp. 137-158.

9. T. N. L. Patterson, “The optimal addition of points to quadrature formulae," Math. Comp., v. 22, 1968, pp. 847-856.

10. R. Piessens \& M. BRANDERS, "A note on the optimal addition of abscissas to quadrature formulas of Gauss and Lobatto type," Math. Comp., v. 28, 1974, pp. 135-139.

11. P. TURÁN, "On the theory of the mechanical quadrature," Acta Sci. Math. (Szeged), v. 12, 1950, pp. 30-37. 